\title{
Probing isotope effects in chemical reactions using single ions
}

\author{
Peter F. Staanum, ${ }^{1,2}$ Klaus Højbjerre, ${ }^{1,2}$ Roland Wester, ${ }^{3}$ and Michael Drewsen ${ }^{1,2}$ \\ ${ }^{1}$ QUANTOP - Danish National Research Foundation Centre for Quantum Optics \\ ${ }^{2}$ Department of Physics and Astronomy, University of Aarhus, Denmark \\ ${ }^{3}$ Physikalisches Institut, Universität Freiburg, Hermann-Herder-Strasse 3, 79104 Freiburg, Germany
}

(Dated: October 29, 2018)

\begin{abstract}
Isotope effects in reactions between $\mathrm{Mg}^{+}$in the $3 \mathrm{p}^{2} P_{3 / 2}$ excited state and molecular hydrogen at thermal energies are studied through single reaction events. From only $\sim 250$ reactions with $\mathrm{HD}$, the branching ratio between formation of $\mathrm{MgD}^{+}$and $\mathrm{MgH}^{+}$is found to be larger than 5. From additional 65 reactions with $\mathrm{H}_{2}$ and $\mathrm{D}_{2}$ we find that the overall decay probability of the intermediate $\mathrm{MgH}_{2}^{+}, \mathrm{MgHD}^{+}$or $\mathrm{MgD}_{2}^{+}$complexes is the same. Our study shows that few single ion reactions can provide quantitative information on ion-neutral reactions. Hence, the method is well-suited for reaction studies involving rare species, e.g., rare isotopes or short-lived unstable elements.
\end{abstract}

PACS numbers: 82.30.Fi, 37.10.Pq, 37.10.Mn, 82.20.Kh

Isotope effects often play an important role for the outcome of chemical reactions. For instance the chemical composition of interstellar clouds is strongly influenced by isotope effects in certain reactions [1]. In laboratory experiments, isotope effects observed in isotopic analogs of chemical reactions can provide details about the reaction dynamics. Substitution reactions of the type F+HD and $\mathrm{Cl}+\mathrm{HD}$ are among the simplest reactions where isotope effects can be present. They were already studied when the first chemical lasers were developed, in order to understand the population inversion mechanism and to identify the laser transitions [2]. These studies together with studies of the $\mathrm{F}+\mathrm{D}_{2}$ reaction strongly stimulated the whole field of reactive scattering [3]. Especially the resonance effects observed in the $\mathrm{F}+\mathrm{H}_{2}$ reaction and isotopic analogs [4] have been subject to numerous experimental and theoretical studies finally resulting in a much improved understanding of this benchmark reaction [3, 5]. In another series of experiments, reactions between a beam of ground state atomic ions and $\mathrm{H}_{2}, \mathrm{HD}$ and $\mathrm{D}_{2}$ have been studied. Strong isotope effects have been observed and, e.g., in reactions between alkaline earth ions $\left(\mathrm{X}^{+}\right)$and $\mathrm{HD}$, it was found that $\mathrm{XD}^{+}$formation is preferred for some alkaline earth ions, while $\mathrm{XH}^{+}$ formation is preferred for others [6, 7].

Within the last few years, new techniques have emerged for ion-neutral reaction studies, e.g., of cold reactive collisions [8], single ion reactions [9] as well as reaction dynamics by applying crossed molecular beam imaging [10]. These techniques open up new possibilities in the field of ion-neutral reactive scattering.

In this Letter, we investigate isotope effects in reactions of single ions with the isotopologues of molecular hydrogen, a model system for ion-neutral reactions. More specifically, we consider reactions at thermal energies with $\mathrm{Mg}^{+}$in the $3 \mathrm{p}^{2} P_{3 / 2}$ excited state (excitation energy of $4.4 \mathrm{eV}$ ). Due to the simple internal structure of the reaction partners, the studied reactions represent a simple test case for reaction dynamics involving an elec-

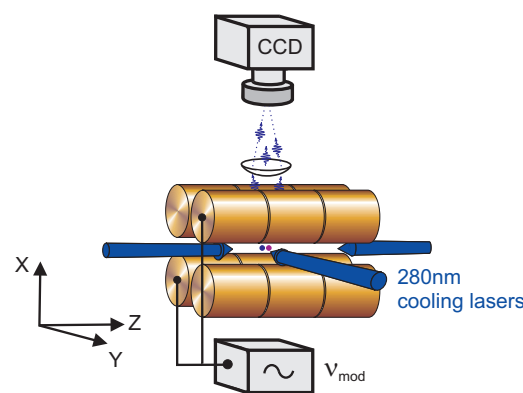

FIG. 1: Sketch of the experimental setup for non-destructive identification of molecular ions. See text for details.

tronically excited atomic collision partner. We make use of an experimental technique with almost $100 \%$ efficiency in detecting single reaction events [9]. With a total of only about 300 reactions, the branching ratio between the reactions

$$
\begin{aligned}
\mathrm{Mg}^{+}\left(3 \mathrm{p}^{2} \mathrm{P}_{3 / 2}\right)+\mathrm{HD} & \rightarrow \mathrm{MgD}^{+}+\mathrm{H} \\
& \rightarrow \mathrm{MgH}^{+}+\mathrm{D}
\end{aligned}
$$

has been found to be larger than 5 . In reactions with $\mathrm{H}_{2}$, $\mathrm{HD}$ and $\mathrm{D}_{2}$ the decay paths leading to either $\mathrm{MgH}^{+}$ or $\mathrm{MgD}^{+}$formation from a $\mathrm{MgH}_{2}^{+}, \mathrm{MgHD}^{+}$or $\mathrm{MgD}_{2}^{+}$ complex have been found to be equally likely within statistical uncertainties. Our experiments demonstrate the prospects for similar single molecular ion studies, e.g., using state prepared molecular ions 11], more complex molecular ions 12, 13] or of astrophysically relevant reactions [14, 15]. The high detection efficiency can furthermore be useful for studies of reactions involving ions of rare species, e.g., superheavy elements [16].

In our study we use a linear Paul trap setup which is described in detail in Ref. [17]. Briefly, as shown in Fig.1, the trap consists of four cylindrical rods, each sectioned into three parts. By applying suitable ac and dc voltages (not indicated in Fig. 11) a harmonic confining potential is created with oscillation frequencies $\omega_{x} \approx \omega_{y}>\omega_{z}$ 
along the $\mathrm{x}-, \mathrm{y}-$ and $\mathrm{z}$-axes, respectively. ${ }^{26} \mathrm{Mg}^{+}$ions are loaded into the trap by crossing an effusive beam of $\mathrm{Mg}$ atoms with a laser beam at $285 \mathrm{~nm}$ in the trap center for resonance-enhanced isotope selective two-photon ionization of ${ }^{26} \mathrm{Mg}$ [18, 19]. The ${ }^{26} \mathrm{Mg}^{+}$ions are Doppler laser cooled on the $3 \mathrm{~s}^{2} S_{1 / 2}-3 \mathrm{p}^{2} P_{3 / 2}$ transition near $280 \mathrm{~nm}$. Individual ions are observed by imaging light, emitted spontaneously during the laser cooling process, onto a charge-coupled device (CCD) camera.

Reactions with $\mathrm{HD}, \mathrm{H}_{2}$ or $\mathrm{D}_{2}$ molecules are investigated by first leaking the gasses into the trap chamber until a steady-state pressure of about $10^{-9}$ Torr is reached, and then loading two ${ }^{26} \mathrm{Mg}^{+}$ions into the trap. Reactions exclusively take place with ${ }^{26} \mathrm{Mg}^{+}$ions excited to the $3 \mathrm{p}^{2} P_{3 / 2}$ state in the laser cooling process, since reactions between ${ }^{26} \mathrm{Mg}^{+}$ions in the 3 s ground state and thermally excited hydrogen molecules are not energetically allowed [20]. After a reaction, the formed molecular ion stays trapped since its acquired kinetic energy only amounts to a small fraction of the trap depth $(\sim 1$ $\mathrm{eV}$ ). Within tens of milliseconds after a single molecular ion is formed, it is sympathetically cooled through the Coulomb interaction with the remaining laser cooled ${ }^{26} \mathrm{Mg}^{+}$ion to form a two-ion Coulomb crystal. Although the molecular ion does not emit light, its presence is evident from the fact that the remaining laser cooled ${ }^{26} \mathrm{Mg}^{+}$ ion is located at one of the two initial ${ }^{26} \mathrm{Mg}^{+}$ion positions (see Fig. 2).

For unambiguous identification of the molecular ion species, its mass is determined by applying the identification technique demonstrated for $\mathrm{CaO}^{+}$ions in Ref. [9]. In short, the method relies on exciting the center-of-mass (CM) mode of the cold two-ion system along the $z$-axis by applying a voltage oscillating at a frequency $\nu_{\text {mod }}$ to two of the end-electrodes as shown in Fig. 1, When $\nu_{\text {mod }}$ is equal to the eigenfrequency of the CM mode, the motion of the ions is resonantly excited. This excitation is clearly visible in the CCD-images as a smearing-out of the fluorescence light from the ${ }^{26} \mathrm{Mg}^{+}$ion along the $z$ axis (see Fig. 2) due to the long exposure time (100 ms) compared to the CM mode oscillation period (typically $\sim 10 \mu \mathrm{s})$. Since the CM eigenfrequency depends on the masses of both ions, the mass of the reaction product ion can now be determined [9].

In practice, the modulation frequency is scanned in five steps through a $100 \mathrm{~Hz}$ narrow frequency interval around $\nu_{m}$, where $\nu_{m}$ denotes the CM mode eigenfrequency for one ${ }^{26} \mathrm{Mg}^{+}$ion and one singly-charged ion of mass $m$, and at each step a CCD image is recorded. Such images are shown in Fig. 2 for one ${ }^{26} \mathrm{Mg}^{+}$ion trapped simultaneously with another ${ }^{26} \mathrm{Mg}^{+}$ion, a ${ }^{26} \mathrm{MgH}^{+}$ion and a ${ }^{26} \mathrm{MgD}^{+}$ion while modulation frequencies of $\nu_{26}, \nu_{27}$ and $\nu_{28}$ are applied. In the experiments, modulation frequencies $\nu_{28}, \nu_{27}, \nu_{26}, \nu_{25}$ and $\nu_{24}$ are applied repeatedly until one ${ }^{26} \mathrm{Mg}^{+}$ion has reacted and the reaction product has been identified. This product ion was ${ }^{26} \mathrm{MgD}^{+}$

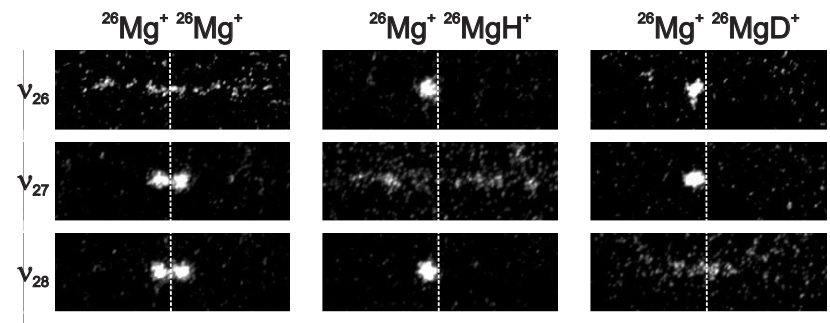

FIG. 2: CCD images of two-ion Coulomb crystals with modulation applied at frequencies $\nu_{26}, \nu_{27}$ and $\nu_{28}$ for identification of ${ }^{26} \mathrm{MgH}^{+}$and ${ }^{26} \mathrm{MgD}^{+}$ions. The dashed vertical lines indicate the position of the trap center along the $\mathrm{z}$-axis.

or ${ }^{26} \mathrm{MgH}^{+}$in all but two cases where a ${ }^{24} \mathrm{Mg}^{+}$ion produced in a charge exchange collision was observed (as in Ref. [19] for calcium). The choice of working with the less abundant ${ }^{26} \mathrm{Mg}^{+}$ion instead of the dominant ${ }^{24} \mathrm{Mg}$ isotope ( $80 \%$ natural abundance) was made to avoid problems in distinguishing ${ }^{24} \mathrm{MgH}^{+}$and ${ }^{24} \mathrm{MgD}^{+}$ions from ${ }^{25} \mathrm{Mg}^{+}$and ${ }^{26} \mathrm{Mg}^{+}$ions, respectively, formed in charge exchange collisions with background gas Mg atoms [19]. At the applied molecular gas pressure $\left(\sim 10^{-9}\right.$ Torr $)$, the $\mathrm{Mg}^{+}$-molecule reaction rate is less than one per minute, which means that the above identification procedure can in general be applied before both of the initially loaded atomic ions have reacted. After a reaction product has been identified the trap is emptied, two new ${ }^{26} \mathrm{Mg}^{+}$ions are loaded and the experiment repeated.

In our study of the branching ratio between reactions (1) and (2), the background pressure of $\mathrm{H}_{2}$ is accounted for by measuring the number of ${ }^{26} \mathrm{MgD}^{+}$ions, $N_{\mathrm{MgD}^{+}}$, and ${ }^{26} \mathrm{MgH}^{+}$ions, $N_{\mathrm{MgH}^{+}}$, formed at four different ratios of the partial pressure of $\mathrm{HD}$ and $\mathrm{H}_{2}, P_{\mathrm{HD}} / P_{\mathrm{H}_{2}}$. The obtained results are presented in Fig. 3 and show an increase of $\mathrm{MgD}^{+}$formation with increased HD pressure.

To understand quantitatively the branching ratio between reactions (11) and (2), we model the reactions as two-step processes. In the first step the neutral molecule is assumed to be captured by the ${ }^{26} \mathrm{Mg}^{+}$ion at long range to form a $\mathrm{MgH}_{2}^{+}$or $\mathrm{MgHD}^{+}$collision complex and, in the second step, a stable $\mathrm{MgH}^{+}$or $\mathrm{MgD}^{+}$ion is formed from this complex at short range. The capture crosssection for the first step is for the present collision energies well approximated by the Langevin capture cross section $\sigma=\left(e / 2 \epsilon_{0}\right) \sqrt{\alpha /\left(\mu v^{2}\right)}[21]$, where $\alpha$ is the polarizability of the neutral molecule, $\mu$ is the reduced mass, and $v$ is the relative velocity in the center-of-mass system. Since the polarizability of $\mathrm{H}_{2}$ and $\mathrm{HD}$ is equal within $1 \%$ [22], in our model we assume $\alpha$ to be identical for $\mathrm{H}_{2}$ and HD. Due to the low ion oscillation frequencies in the trap, the reduced masses are not significantly different from the free-free reaction cases.

The two-step model is valid since the capture range in the first step is of the order of $(\sigma / \pi)^{1 / 2} \sim 6 \AA$ which is much larger than the extension of the hydrogen molecule 


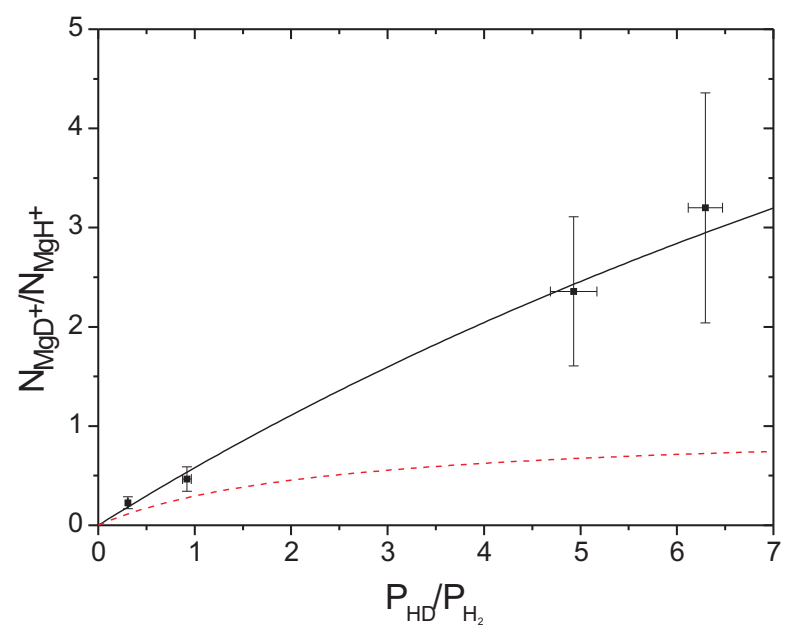

FIG. 3: The ratio between the number of formed ${ }^{26} \mathrm{MgD}^{+}$ and ${ }^{26} \mathrm{MgH}^{+}$ions vs. the relative pressure of $\mathrm{HD}$ and $\mathrm{H}_{2}$ gas. From left to right the data points correspond to $N_{\mathrm{MgD}^{+}}=17$, 21, 33, and 32 and $N_{\mathrm{MgH}^{+}}=75,45,14$, and 10 . The error bars represent statistical uncertainties. A fit of Eq. (3) to the data results in $\eta_{\mathrm{MgD}^{+}}^{\mathrm{HD}} / \eta_{\mathrm{MgH}^{+}}^{\mathrm{H}_{2}}=0.73 \pm 0.17$ and $\eta_{\mathrm{MgH}^{+}}^{\mathrm{HD}} / \eta_{\mathrm{MgH}^{+}}^{\mathrm{H}_{2}}=0.06_{-0.06}^{+0.10}$ (black solid line). The red dashed line represents Eq. (3) in the absence of isotope effects, i.e. with $\eta_{\mathrm{MgD}^{+}}^{\mathrm{HD}} / \eta_{\mathrm{MgH}^{+}}^{\mathrm{H}_{2}}=\eta_{\mathrm{MgH}^{+}}^{\mathrm{HD}} / \eta_{\mathrm{MgH}^{+}}^{\mathrm{H}_{2}}=0.5$.

and the $\mathrm{Mg}^{+}$ion as well as the de Broglie wavelength relevant for the reaction. If the probability for molecule formation in the second step is denoted by $\eta$, the molecular ion formation rate $\Gamma$ is then given by $p_{\text {exc }} \times n \times v \times \sigma \times \eta$, where $p_{\text {exc }}$ is the ${ }^{26} \mathrm{Mg}^{+}$excitation probability and $n$ is the neutral molecule density. Using that density is proportional to pressure we find that

$$
\begin{aligned}
& \frac{N_{\mathrm{MgD}^{+}}}{N_{\mathrm{MgH}^{+}}}=\frac{\Gamma_{\mathrm{MgD}^{+}}}{\Gamma_{\mathrm{MgH}^{+}}} \\
& =\frac{\left(\eta_{\mathrm{MgD}^{+}}^{\mathrm{HD}} / \eta_{\mathrm{MgH}^{+}}^{\mathrm{H}_{2}}\right) \times\left(P_{\mathrm{HD}} / P_{\mathrm{H}_{2}}\right)}{\sqrt{\mu_{\mathrm{HD}} / \mu_{\mathrm{H}_{2}}}+\left(\eta_{\mathrm{MgH}^{+}}^{\mathrm{HD}} / \eta_{\mathrm{MgH}^{+}}^{\mathrm{H}}\right) \times\left(P_{\mathrm{HD}} / P_{\mathrm{H}_{2}}\right)},
\end{aligned}
$$

where $\eta_{M^{+}}^{M^{\prime}}$ is the efficiency of $M^{+}\left(\mathrm{MgH}^{+}\right.$or $\left.\mathrm{MgD}^{+}\right)$ formation after reaction with $M^{\prime}\left(\mathrm{H}_{2}\right.$ or $\left.\mathrm{HD}\right)$. This expression is independent of $p_{\text {exc }}$ and of the absolute partial pressures, which greatly reduces systematic errors. The relative partial pressures are measured with a restgas analyzer and for all values of the ratio $P_{\mathrm{HD}} / P_{\mathrm{H}_{2}}$ we estimate its systematic uncertainty to be less than $25 \%$. From the parameters determined from the fit (see Fig. 3), the branching ratio $\eta_{\mathrm{MgD}^{+}}^{\mathrm{HD}} / \eta_{\mathrm{MgH}^{+}}^{\mathrm{HD}}$ can be determined. Taking the statistical uncertainties into account, we find that the branching ratio is unbound from above and has a lower limit of 5 , thus demonstrating a dramatic intramolecular isotope effect. In addition, the ratio $\left(\eta_{\mathrm{MgH}^{+}}^{\mathrm{HD}}+\eta_{\mathrm{MgD}^{+}}^{\mathrm{HD}}\right) / \eta_{\mathrm{MgH}^{+}}^{\mathrm{H}_{2}}$ is consistent with unity which indicates that the probability of forming a mag-

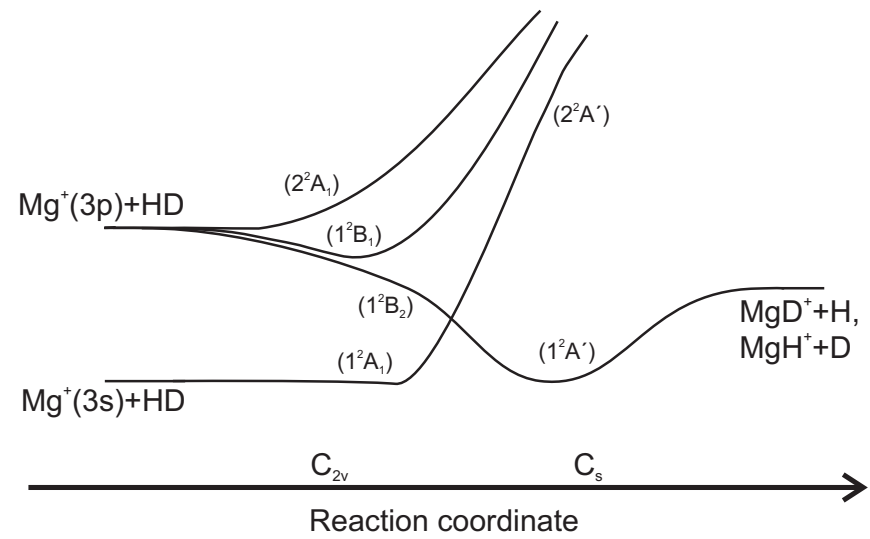

FIG. 4: Sketch of relevant potential surfaces for the $\mathrm{Mg}^{+}+\mathrm{HD}$ reaction [23, 24]. On the left (right) hand side the potential surfaces are labelled according to $\mathrm{C}_{2 v}\left(\mathrm{C}_{s}\right)$ symmetry. As discussed in the text, the reaction probably proceeds by insertion of $\mathrm{Mg}^{+}$into the HD bond on the $1^{2} B_{2}$ potential surface, followed by $\mathrm{Mg}^{+}-\mathrm{D}$ or $\mathrm{Mg}^{+}-\mathrm{H}$ bond formation.

nesium hydride ion is equally large from any of the two complexes $\mathrm{MgH}_{2}^{+}$or $\mathrm{MgHD}^{+}$.

In a second series of measurements we studied reactions between $\mathrm{Mg}^{+}$and a mixture of $43 \pm 2 \% \mathrm{H}_{2}$ and $57 \pm 2 \% \mathrm{D}_{2}$ gas. We observed the production of $40 \mathrm{MgH}^{+}$ ions and $25 \mathrm{MgD}^{+}$ions. Using the same two-step model as above we find that $\eta_{\mathrm{MgH}^{+}}^{\mathrm{H}_{2}} / \eta_{\mathrm{MgD}^{+}}^{\mathrm{D}_{2}}=1.5 \pm 0.4$.

Substitution reactions between a ${ }^{26} \mathrm{MgH}^{+}$or ${ }^{26} \mathrm{MgD}^{+}$ ion and a $\mathrm{H}_{2}$, $\mathrm{HD}$ or $\mathrm{D}_{2}$ molecule could potentially give rise to a systematic error. Such reactions were, however, only observed on two occasions (one $\mathrm{MgH}^{+}+\mathrm{D}_{2} \rightarrow$ $\mathrm{MgD}^{+}+\mathrm{H}+\mathrm{D}$, one $\left.\mathrm{MgD}^{+}+\mathrm{H}_{2} \rightarrow \mathrm{MgH}^{+}+\mathrm{H}+\mathrm{D}\right)$ in a period of more than 20 minutes and hence they do not give rise to systematic errors on the results presented here.

The most striking of the above results is the strong intramolecular isotope effect in reactions (11) and (2). This finding cannot be explained by a simple statistical model based on an assumption of an equal probability for populating energetically accessible states of ${ }^{26} \mathrm{MgH}^{+}$and ${ }^{26} \mathrm{MgD}^{+}$. This assumption only gives rise to $\eta_{\mathrm{MgD}^{+}}^{\mathrm{HD}} / \eta_{\mathrm{MgH}^{+}}^{\mathrm{HD}} \sim 2$ and therefore we attribute the observed isotope effect to a dynamical mechanism. In the ion beam experiments of Ref. [6] a similar isotope effect has been observed in reactions between ground state $\mathrm{Mg}^{+}$ ions and HD molecules at center-of-mass energies up to $11 \mathrm{eV}$. This was rationalized in terms of an impulsive interaction with a thermodynamic threshold.

A schematic view of the potential surfaces involved in the reaction is shown in Fig. 4. The analogous potential surfaces for $\mathrm{MgD}_{2}^{+}$was explored in a photofragmentation study where $\mathrm{MgD}^{+}$formation was observed after laser excitation from the $1^{2} A_{1}$ state to the red of the $\mathrm{Mg}^{+}(3 \mathrm{p})+\mathrm{D}_{2}$ asymptote 25]. From the observed $\mathrm{MgD}^{+}$ spectrum it was argued that $\mathrm{MgD}^{+}$is formed by direct 
and fast reactions on the $1^{2} B_{2}$ surface in $\mathrm{C}_{2 v}$ geometry through a bond-stretch mechanism as well as from the $1^{2} B_{1}$ state, possibly through a coupling to the $1^{2} B_{2}$ state. On the $1^{2} B_{2}$ potential surface the $\mathrm{Mg}^{+}$ion becomes inserted in the $\mathrm{D}_{2}$ bond such that the D-D bond is stretched and eventually broken and a $\mathrm{Mg}^{+}-\mathrm{D}$ bond is formed 23, 25]. In addition, in this study no dissociation into $\mathrm{Mg}^{+}(3 \mathrm{~s})+\mathrm{D}_{2}$ was observed. In our two-step model this corresponds to the values of $\eta_{\mathrm{MgH}^{+}}^{\mathrm{H}_{2}}, \eta_{\mathrm{MgD}^{+}}^{\mathrm{D}_{2}}$ and $\left(\eta_{\mathrm{MgD}^{+}}^{\mathrm{HD}}+\eta_{\mathrm{MgH}^{+}}^{\mathrm{HD}}\right)$ being close to unity, which indeed is in good agreement with the $\mathrm{Mg}^{+}+\mathrm{H}_{2}$ reaction rate measured in a previous study [20, 26].

The investigation of photofragmentation indicate that the $\mathrm{Mg}^{+}+\mathrm{HD}$ reaction discussed in the present paper proceeds via the $1^{2} B_{2}$ surface through a bondstretch mechanism that eventually favors the formation of $\mathrm{MgD}^{+}$[23, 25]. To fully understand the transition from a $\mathrm{MgHD}^{+}$complex to a potential surface favoring the $\mathrm{MgD}^{+}+\mathrm{H}$ asymptote rather than the $\mathrm{MgH}^{+}+\mathrm{D}$ asymptote requires a detailed theoretical study. It might be necessary to consider the details of the conical intersection which arises from the crossing of the $1^{2} \mathrm{~A}_{1}$ and $1^{2} \mathrm{~B}_{2}$ potential surfaces. Non-adiabatic couplings at the conical intersection could give rise to a preference of the $\mathrm{MgD}^{+}$channel over the $\mathrm{MgH}^{+}$channel. The same mechanism could be responsible for the isotope effect observed in reactions with ground state $\mathrm{Mg}^{+}$ions [6]

In conclusion, we have found that reactions between $\mathrm{Mg}^{+}$in the $3 \mathrm{p}^{2} P_{3 / 2}$ excited state and $\mathrm{HD}$ molecules at thermal energies preferentially leads to the formation of $\mathrm{MgD}^{+}$rather than $\mathrm{MgH}^{+}$with a branching ratio larger than 5. Additional reactions with $\mathrm{H}_{2}$ and $\mathrm{D}_{2}$ have shown that after a reaction complex has formed through a Langevin capture process, the molecular ion formation efficiencies after capture of a $\mathrm{HD}, \mathrm{H}_{2}$ or $\mathrm{D}_{2}$ molecule are equal within statistical uncertainties. The efficiencies are consistent with unity which is in agreement with observations in a previous study of $\mathrm{MgD}_{2}^{+}$photofragmentation [25]. Our measurements demonstrate that it is possible to determine quantitatively the branching ratios and relative reaction rates in ion-neutral reactions by observing only a few hundred single reactions. The method should in the future be applicable to a variety of studies, e.g., of astrophysically relevant reactions, chemistry of superheavy elements and reactions involving more complex molecules.

The authors are grateful to Jeppe Olsen and Lorenz Cederbaum for fruitful discussions. PFS acknowledges support from the Danish Natural Science Research Council. RWs visit in Århus was supported by the European Science Foundation through the CATS network.
[2] P. H. Corneil and G. C. Pimentel, J. Chem. Phys. 49, 1379 (1968); M. J. Berry, ibid. 59, 6229 (1973).

[3] W. Hu and G. C. Schatz, J. Chem. Phys 125, 132301 (2006), and references therein.

[4] D. M. Neumark, A. M. Wodtke, G. N. Robinson, C. C. Hayden, and Y. T. Lee, Phys. Rev. Lett. 53, 226 (1984).

[5] M. Qui, Z. Ren, L. Che, D. Dai, S. Harich, X. Wang, X. Yang, C. Xu, D. Xie, M. Gustafsson, et al., Science 311, 1440 (2006).

[6] N. Dalleska, K. Crellin, and P. Armentrout, J. Phys. Chem. 97, 3123 (1993).

[7] R. Georgiadis and P. Armentrout, J. Phys. Chem. 92, 7060 (1988).

[8] S. Willitsch, M. T. Bell, A. D. Gingell, S. R. Procter, and T. P. Softley, Phys. Rev. Lett. 100, 043203 (2008).

[9] M. Drewsen, A. Mortensen, R. Martinussen, P. Staanum, and J. L. Sørensen, Phys. Rev. Lett. 93, 243201 (2004).

[10] J. Mikosch, S. Trippel, C. Eichhorn, R. Otto, U. Lourderaj, J. X. Zhang, W. L. Hase, M. Weidemüller, and R. Wester, Science 319, 183 (2008).

[11] I. S. Vogelius, L. B. Madsen, and M. Drewsen, Phys. Rev. Lett. 89, 173003 (2002); Phys. Rev. A 70, 053412 (2004).

[12] A. Ostendorf, C. B. Zhang, M. A. Wilson, D. Offenberg, B. Roth, and S. Schiller, Phys. Rev. Lett. 97, 243005 (2006).

[13] K. Højbjerre, D. Offenberg, C. Z. Bisgaard, H. Stapelfeldt, P. Staanum, A. Mortensen, and M. Drewsen, to appear in Phys. Rev. A, Rapid Communications.

[14] D. Gerlich, E. Herbst, and E. Roueff, Planetary and Space Science 50, 1275 (2002).

[15] S. Trippel, J. Mikosch, R. Berhane, R. Otto, M. Weidemüller, and R. Wester, Phys. Rev. Lett. 97, 193003 (2006).

[16] M. Drewsen, Eur. Phys. J. D 45, 125 (2007); M. Schädel, ibid. 45, 67 (2007); H. Haba, ibid. 45, 81 (2007).

[17] M. Drewsen, I. Jensen, J. Lindballe, N. Nissen, R. Martinussen, A. Mortensen, P. Staanum, and D. Voigt, Int. J. Mass. Spect. 229, 83 (2003).

[18] N. Kjærgaard, L. Hornekær, A. Thommesen, Z. Videsen, and M. Drewsen, Appl. Phys. B 71, 207 (2000).

[19] A. Mortensen, J. Lindballe, I. Jensen, P. Staanum, D. Voigt, and M. Drewsen, Phys. Rev. A 69, 042502 (2004).

[20] K. Mølhave and M. Drewsen, Phys. Rev. A 62, 011401(R) (2000).

[21] R. D. Levine, Molecular Reaction Dynamics (Cambridge University Press, Cambridge, 2005).

[22] W. Kolos and L. Wolniewicz, J. Chem. Phys. 46, 1426 (1966).

[23] P. D. Kleiber and J. Chen, Int. Rev. Phys. Chem. 17, 1 (1998).

[24] C. W. Bauschlicher Jr., Chem. Phys. Lett. 201, 11 (1993).

[25] L. N. Ding, M. A. Young, P. D. Kleiber, and W. C. Stwalley, J. Phys. Chem. 97, 2181 (1993).

[26] K. Mølhave, Masters thesis, University of Aarhus (2000). 